\title{
On the role of the Prandtl number in convection driven by heat sources and sinks
}

\author{
Benjamin Miquel ${ }^{1} \dagger$, Vincent Bouillaut ${ }^{1}$, Sébastien Aumaître ${ }^{1}$, and \\ Basile Gallet ${ }^{1}$
}

${ }^{1}$ Université Paris-Saclay, CEA, CNRS, SPEC, 91191, Gif-sur-Yvette, France

(Received xx; revised $\mathrm{xx}$; accepted $\mathrm{xx}$ )

\begin{abstract}
We report on a numerical study of turbulent convection driven by a combination of internal heat sources and sinks. Motivated by a recent experimental realisation Lepot et al. 2018), we focus on the situation where the cooling is uniform, while the internal heating is localised near the bottom boundary, over approximately one tenth of the domain height. We obtain scaling laws $N u \sim R a^{\gamma} \operatorname{Pr}^{\chi}$ for the heat transfer as measured by the Nusselt number $N u$ expressed as a function of the Rayleigh number $R a$ and the Prandtl number $\operatorname{Pr}$. After confirming the experimental value $\gamma \approx 1 / 2$ for the dependence on $R a$, we identify several regimes of dependence on $P r$. For a stress-free bottom surface and within a range as broad as $\operatorname{Pr} \in[0.003,10]$, we observe the exponent $\chi \approx 1 / 2$, in agreement with Spiegel's mixing length theory. For a no-slip bottom surface we observe a transition from $\chi \approx 1 / 2$ for $\operatorname{Pr} \leqslant 0.04$ to $\chi \approx 1 / 6$ for $\operatorname{Pr} \geqslant 0.04$, in agreement with scaling predictions by Bouillaut et al. The latter scaling regime stems from heat accumulation in the stagnant layer adjacent to a no-slip bottom boundary, which we characterise by comparing the local contributions of diffusive and convective thermal fluxes.
\end{abstract}

Key words: convective flows, turbulence

\section{Introduction}

Thermal forcing is an important driver for turbulence in numerous natural and industrial flows. Buoyancy greatly impacts the oceanic dynamics of the Earth (Sutherland et al. 2019) and of ice-clad moons (Miquel et al. 2018; Soderlund 2019), atmospheric flows (Yano et al. 2003), and internal dynamics of planets and stars (Aurnou et al. 2015; Guervilly et al. 2019). Implications and consequences range from climate modelling (Plant \& Yano 2015) to the generation of large scale magnetic field via the dynamo effect (Browning 2008; Soderlund et al. 2012; Calkins et al. 2015), to cite a few. A global characterisation of such convective flows boils down to the critical issue of turbulent heat transport: How is the enhanced heat flux related to the temperature inhomogeneities, the geometry of the system and the properties of the fluid? Owing to the extremely turbulent nature of the natural and industrial flows of interest, one tries to answer the questions above by identifying scaling-laws that capture the essential mechanisms at play and are amenable to extrapolations.

Here we study Convection driven by Internal Sources and Sinks (CISS), whose scaling behavior departs from the standard setups of thermal convection, such as the

$\dagger$ Email address for correspondence: benjamin.miquel@cea.fr 
Rayleigh-Bénard convection (RBC) (Ahlers et al. 2009; Chillà \& Schumacher 2012), horizontal convection (HC) (Vreugdenhil et al. 2017; Rocha et al.|2020), or even internallyheated convection (IHC) with a cooling top surface (Goluskin 2015) or between fixedtemperature plates (Goluskin \& van der Poel 2016). Indeed, the latter setups all display thermal boundary layers that greatly throttle the heat flux, whereas these boundary layers are bypassed in CISS, which results in a more efficient heat transport scaling regime. For instance, a recent experimental and numerical study of CISS in water by Lepot et al. (2018) investigated the dependence of the heat flux with the temperature difference in the fluid. The dimensionless counterpart to this question amounts to finding a relationship between the standard Nusselt number and Rayleigh number (see equation 2.3 for definitions). Lepot et al. (2018) measured $N u \sim R a^{\gamma}$ with $\gamma \approx 0.5$. This is in strong contrast with RBC experiments and numerical studies where $0.27 \leqslant$ $\gamma \leqslant 0.39$ Castaing et al. 1989; Chavanne et al. 1997; Niemela et al. 2000; Ahlers et al. 2009; He et al. 2012; Doering et al. 2019). This range of measured exponents support the fact that diffusivity coefficients play a role in the RBC setup, through the formation of boundary layers that throttle the heat flux.

The scope of the present study is to investigate the variation of the heat flux as the Prandtl number (defined as the ratio of the fluid diffusivity coefficients, see eq. 2.2) varies. Even for the much studied RBC case, such studies remain scarce. In the laboratory, low Prandtl numbers are reached by employing liquid metal (Fauve et al. 1981; Cioni et al. 1997; Aurnou et al. 2018), which severely hinders measurements and virtually forbids visualizations. Direct numerical simulations (DNS) exhibit much more flexibility from the standpoint of diffusivity coefficients and have proved invaluable for understanding the role on the Prandtl number for both RBC (Shishkina et al. 2017) and HC (Shishkina \& Wagner 2016). In the present study, we employ DNS to explore the parameter space of CISS. In particular, we put to scrutiny recent predictions by Bouillaut et al. (2019) that not only provide a model for the experimental results of Lepot et al. (2018), but also predict two distinct regimes characterized by different scaling behaviours with respect to the Prandtl number.

The paper is laid out as follows. The formulation of the governing equations and the numerical methods are presented in section 2. Models for the heat transfer law are presented in section 3 and the existence of two different regimes is predicted, depending on the Prandtl number. These regimes are evidenced in a data set obtained from direct numerical simulations (DNS) and presented in section 4 together with an analysis of the thermal boundary layers. Finally, section 5 contains a discussion and closing remarks.

\section{Governing equations and numerical procedure}

\subsection{Model and parameters}

We consider a horizontal fluid layer of depth $H$, kinematic viscosity $\nu$, thermal diffusivity $\kappa$, specific heat capacity $C_{p}$, and thermal expansion coefficient $\alpha$, all of which are considered constant. The layer is subjected to the vertical gravitational acceleration of (uniform) magnitude $g$. We consider the Boussinesq approximation, where the density $\rho$ is constant and uniform, except in the expression of the buoyancy force. Volumic heat sources and sinks are present in the bulk of the fluid. In the experiment by Lepot et al. (2018) and Bouillaut et al. (2019), a powerful spotlight shines visible light through the transparent bottom of the tank, into dyed water where absorption occurs over a typical length $\ell$. Following Beer-Lambert's law, the intensity of light decreases exponentially with the height $Z$, measured from the bottom surface. Thus, we model the experimental 
On the role of the Prandtl number in convection driven by heat sources and sinks 3 radiation-induced heating with an exponentially decreasing volumic heat-source in the present numerical work:

$$
Q_{L}(Z)=\frac{P}{\ell} \frac{\exp (-Z / \ell)}{1-\exp (-H / \ell)},
$$

where $P$ is the incident light flux at the bottom of the tank (power per unit area), which gets deposited into the fluid through absorption. In the laboratory experiment, this internal heat source yields "secular heating" of the fluid, which amounts to a quasi-stationary state with homogeneous internal cooling $Q_{c}(Z)=P / H$ balancing the radiative heat input, so that the net power deposited in the fluid layer vanishes: $\int_{0}^{H}\left(Q_{L}(Z)-Q_{c}(Z)\right) \mathrm{d} Z=0$. We stress the fact that, motivated by the experimental realisations, we are concerned with a distribution of heat sources that takes on finite values at the boundaries. In that respect, the problem of interest here crucially differs from previous studies by Barker et al. (2014), who considers a distribution of heat sources and sinks that vanishes at the boundaries.

The characteristics of the setup can be cast into three dimensionless control parameters, namely the Prandtl number $\operatorname{Pr}$, the flux-based Rayleigh number $R a_{Q}$, and the dimensionless light absorption-length $\widetilde{\ell}$ :

$$
\operatorname{Pr}=\frac{\nu}{\kappa} ; \quad R a_{Q}=\frac{\alpha g P H^{4}}{\rho C_{p} \kappa^{2} \nu} ; \quad \tilde{\ell}=\frac{\ell}{H} .
$$

The response of the fluid to the imposed flux may be characterised by the magnitude of the temperature difference $\Delta T$ that appears in the flow, the characteristic velocity of which is $U$. To make contact with the numerous RBC literature, one can then define the Reynolds number $R e$, the temperature-based Rayleigh number $R a$, and the Nusselt number $N u$ :

$$
R e=\frac{U H}{\nu} ; \quad R a=\frac{\alpha g \Delta T H^{3}}{\nu \kappa} ; \quad N u=\frac{P H}{\rho C_{p} \kappa \Delta T} ; \quad \text { with } \quad R a_{Q}=R a N u .
$$

\subsection{Dimensionless equations and parameters}

The Boussinesq equations are written in a dimensionless form by counting lengths in units of the depth $H$, time in units of diffusive time $H^{2} / \kappa$, and temperature in units of $\nu \kappa / \alpha g H^{3}$. Thus, the governing equations for the solenoidal velocity field $(\boldsymbol{\nabla} \cdot \boldsymbol{u}=0)$ and temperature field $\theta$ are:

$$
\begin{gathered}
\operatorname{Pr}^{-1}\left(\partial_{t} \boldsymbol{u}+\boldsymbol{u} \cdot \boldsymbol{\nabla u}\right)=-\nabla p+\theta \hat{\mathbf{e}}_{z}+\nabla^{2} \boldsymbol{u}, \\
\partial_{t} \theta+\boldsymbol{u} \cdot \nabla \theta=\nabla^{2} \theta+R a_{Q}\left(\frac{\exp (-z / \tilde{\ell})}{\tilde{\ell}[1-\exp (-1 / \tilde{\ell})]}-1\right),
\end{gathered}
$$

where $z=Z / H$. These governing equations are supplemented with adiabatic boundary conditions for temperature: $\partial_{z} \theta=0$ at $z=0,1$. Kinematic boundary conditions are always impenetrable $(w=0)$ and either no-slip $(u=v=0)$ or stress-free $\left(\partial_{z} u=\partial_{z} v=0\right)$ at top and bottom. Finally, we consider periodic boundary conditions in the horizontal.

\subsection{Numerical procedure}

Direct numerical simulations (DNS) of equations (2.4) are performed with the HPC code Coral, which employs a Chebyshev-Fourier-Fourier decomposition in space and a family of implicit-explicit timestepping schemes. The boundary conditions in the vertical direction $z$ are enforced using Galerkin basis recombination. Computations are initialised 
with either small-amplitude noise or a previous solution and are carried out until a statistically stationary regime is clearly identified. Coral was already used for studying CISS in Miquel et al. (2019), where it is benchmarked against analytical solutions. The non-periodic version of Coral has also been benchmarked against the numerical solutions obtained by Lepot et al. (2018) using the solver Dedalus (Burns et al. 2020), for $\operatorname{Pr}=1$ and 7 .

\section{Scaling arguments}

For completeness, we recall in broad strokes the scalings predicted by Bouillaut et al. (2019). Experiments and numerical simulations indicate that the large-scale flow consists of turbulent convection cells of aspect ratio close to one, the velocity of which obeys the free-fall scaling-law $U \sim \sqrt{\alpha g \Delta T H}$.

\subsection{Bulk transport}

As a fluid element circles around a turbulent convection cell, it rapidly heats up in the region $z \lesssim \widetilde{\ell}$, while it slowly cools down in the region $z \gtrsim \widetilde{\ell}$. In other words, the heat input inside the region $z \lesssim \tilde{\ell}$ is carried outside the heating region by the flow, which amounts to a balance between the source term and the horizontal advection term in the heat equation:

$$
\rho C_{p} U \partial_{x} T \sim \frac{P}{\ell} .
$$

Upon injecting the free-fall velocity estimate, the balance above yields an estimate $\Delta T_{\text {bulk }}$ for the temperature difference between the heating region $z \lesssim \widetilde{\ell}$ and the bulk region $z \gtrsim \widetilde{\ell}$. In dimensional and dimensionless form:

$$
\left(\Delta T_{\text {bulk }}\right)^{3} \sim \frac{P^{2} H}{\alpha g \rho^{2} C_{p}^{2} \ell^{2}} ; \quad N u \sim \widetilde{\ell} \sqrt{\operatorname{RaPr}} .
$$

The reasoning above and the associated $\Delta T_{\text {bulk }}$ do not take into account the velocity boundary conditions at the bottom plate. In the next section, we show that the latter can induce a possibly stronger temperature difference $\Delta T_{\mathrm{BL}}$ between the immediate vicinity of the bottom boundary and the heating region $z \lesssim \tilde{\ell}$. The total temperature difference between the bottom plate and the bulk region $z \gtrsim \tilde{\ell}$ is then estimated as $\Delta T=\Delta T_{\mathrm{BL}}+\Delta T_{\text {bulk }}$.

\subsection{Stagnant layers near no-slip boundaries}

The scaling-law (3.2) may be modified in the case of a no-slip bottom boundary condition. Indeed, the stagnant layer in the immediate vicinity of the bottom plate accumulates heat, and without efficient advection outside this region, the heat can only be diffused away. This leads to a thermal boundary layer of thickness $\delta_{\Theta}$. Balancing the heat source term with the diffusive one estimated inside the boundary layer leads to:

$$
\rho C_{p} \kappa \frac{\Delta T_{\mathrm{BL}}}{\delta_{\Theta}^{2}} \sim \frac{P}{\ell}, \quad \text { yielding : } \quad \Delta T_{\mathrm{BL}}=\frac{P H^{2}}{\rho C_{p} \kappa \ell} \frac{\delta_{\Theta}^{2}}{H^{2}} .
$$

A final relation between the Nusselt number and the Rayleigh and Prandtl numbers is obtained by supplementing (3.3) with a scaling estimate of the boundary layer thickness $\delta_{\Theta}$. The latter depends crucially on the Prandtl number: in the case of low-Prandtl fluids, a naive laminar estimate would lead to a thermal boundary layer thicker than the kinematic one. However, one expects vigorous turbulent mixing outside the kinematic 
On the role of the Prandtl number in convection driven by heat sources and sinks 5

boundary layer, so that both boundary layers end up having the same thickness. We obtain $\delta_{\Theta} \sim H / \sqrt{R e}$. Upon estimating the Reynolds number using the free-fall velocity scaling (based on $\Delta T_{\mathrm{BL}}$ in a worst-case scenario), equation (3.3) becomes:

$$
\left(\Delta T_{\mathrm{BL}}\right)^{3} \sim \frac{P^{2} H}{\alpha g \rho^{2} C_{p}^{2} \ell^{2}} \frac{\nu^{2}}{\kappa^{2}} .
$$

which remains negligible compared with the bulk temperature jump from equation (3.2) when $\operatorname{Pr} \ll 1$. As a consequence, the scaling-law $N u \sim \tilde{\ell} \sqrt{\operatorname{RaPr}}$ remains valid and is not modified by the stagnant bottom layer for $\operatorname{Pr} \ll 1$.

The situation is crucially different for high-Prandtl fluids. The thermal boundary layer is much thinner than, and nested into, the kinetic one. The flow in the vicinity of the no-slip bottom surface is perceived by the thermal boundary layer as a parallel shear flow, the bottom shear $S(0)$ being estimated as $S(0) \sim U / \delta_{\nu} \sim U \sqrt{R e} / H \sim \sqrt{U^{3} / H \nu}$. The boundary-layer thickness is set by a balance between slow advection by the shear, at a speed $S(0) \delta_{\Theta}$, and vertical diffusion:

$$
\rho C_{p} S(0) \delta_{\Theta} \frac{\Delta T_{\mathrm{BL}}}{H} \sim \frac{\rho C_{p} \kappa \Delta T_{\mathrm{BL}}}{\delta_{\Theta}^{2}}, \quad \text { so that } \quad \frac{\delta_{\Theta}}{H} \sim\left(\frac{\kappa}{S(0) H^{2}}\right)^{1 / 3} \sim \frac{1}{\operatorname{Re}^{1 / 2} \operatorname{Pr}^{1 / 3}} .
$$

Injecting this expression for the thermal boundary layer thickness into equation (3.3), one gets the temperature jump associated with the sheared thermal layer and the associated dimensionless heat flux:

$$
\left(\Delta T_{\mathrm{BL}}\right)^{3} \sim \frac{P^{2} H}{\alpha g \rho^{2} C_{p}^{2} \ell^{2}}\left(\frac{\nu}{\kappa}\right)^{2 / 3} ; \quad N u \sim \widetilde{\ell} R a^{1 / 2} \operatorname{Pr}^{1 / 6} .
$$

Noticeably, a comparison with equation (3.2) informs us that the temperature jump in the stagnant boundary layer dominates the temperature jump in the bulk: $\Delta T \simeq \Delta T_{\mathrm{BL}}$ when $\operatorname{Pr} \gg 1$. This observation results in a modification of the Nusselt number scalinglaw due to boundary layer corrections, see equation (3.6).

The last case that requires our attention is the case of high-Prandtl-number flows with a stress-free bottom surface. Within the thermal boundary layer, the flow is well approximated by a uniform parallel flow with velocity $U \sim \sqrt{\alpha g \Delta T_{\mathrm{BL}} H}$. Here, in view of finding hypothetical boundary layer corrections, we assume again that the overall temperature difference is dominated by the contribution from the boundary layer $\Delta T_{\mathrm{BL}}$, before testing this assumption a posteriori. A triple balance between horizontal advection, diffusion through the layer, and heating yields:

$$
\rho C_{p} \sqrt{\alpha g \Delta T_{\mathrm{BL}} H} \frac{\Delta T_{\mathrm{BL}}}{H} \sim \rho C_{p} \kappa \frac{\Delta T_{\mathrm{BL}}}{\delta_{\Theta}^{2}} \sim \frac{P}{\ell} .
$$

This balance is similar to the bulk balance (eq. 3.1). Some straightforward algebra confirms that a transport law identical to (3.2) is found. We conclude that no boundarylayer induced corrections to the heat-transport scaling-law are necessary in this case.

To summarize, the scaling theory results in the following predictions for the dimensionless heat flux:

$$
N u=\left\{\begin{array}{ccc}
c_{1} \tilde{\ell} \sqrt{R a P r} & \text { with stress-free b. c., } & \forall P r, \\
c_{2} \widetilde{\ell} \sqrt{R a P r} & \text { with no-slip b. c., } & \operatorname{Pr} \ll \operatorname{Pr} r_{*}, \\
c_{3} \widetilde{\ell} a^{1 / 2} \operatorname{Pr}^{1 / 6} & \text { with no-slip b. c., } & \operatorname{Pr} \gg P r_{*},
\end{array}\right.
$$

where the dimensionless prefactors $c_{i}$ remain to be determined. The transition Prandtl 

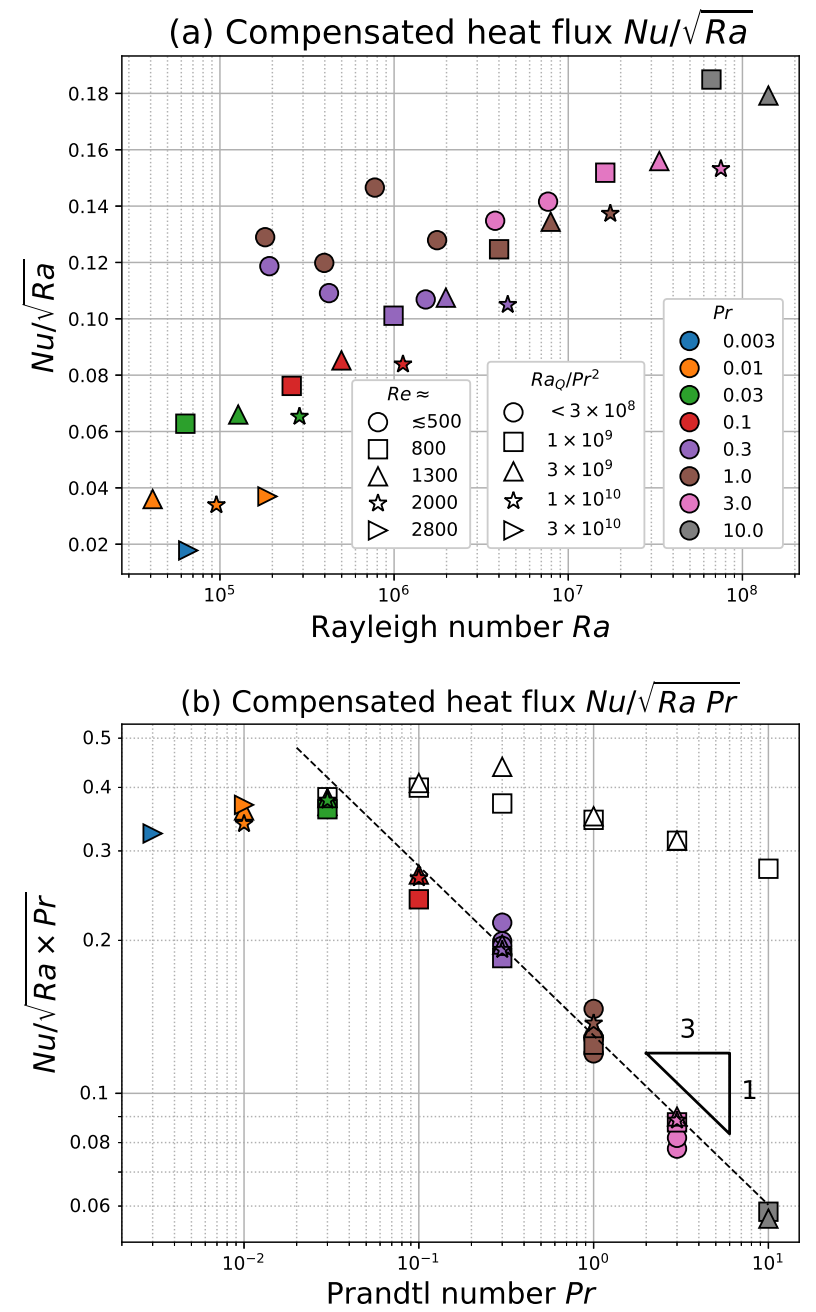

Figure 1. (a) Compensated heat-flux $N u / \sqrt{R a}$ as a function of the Rayleigh number $R a$ for no-slip boundary conditions. The colour codes for the Prandtl number $P r$, while the heating intensity as measured by $R a_{Q} / \mathrm{Pr}^{2}$ is encoded in the shape of the symbols. For convenience, we provide a correspondence between the parameter $R a_{Q} / \mathrm{Pr}^{2}$ and the Reynolds number Re. This colour/shape code is employed consistently thereafter throughout the paper. (b) Compensated heat-flux $N u / \sqrt{R a P r}$ as a function of $\operatorname{Pr}$ for no-slip (filled symbols) and stress-free (open symbols) bottom boundaries.

number $\operatorname{Pr}_{*}$ is estimated by equating the two no-slip predictions at $\operatorname{Pr}=\operatorname{Pr}_{*}$, which yields $P r_{*}=\left(c_{3} / c_{2}\right)^{3}$.

\section{Observations of the two regimes}

We now turn to an extensive numerical study, with the goal of (i) assessing the validity of the scaling predictions (3.8), (ii) estimating the transition Prandtl number $\mathrm{Pr}_{*}$, and (iii) determining the dimensionless prefactors $c_{i}$, so that (3.8) can be used as a quantitative relation in cases of practical interest. Throughout this section, the dimensionless absorption length is set to $\widetilde{\ell}=0.1$. The domain is cubic with periodic 
boundary conditions in the horizontal and insulated top and bottom boundaries. The top boundary condition is always no-slip, and the bottom one is either stress-free or no-slip.

\subsection{Heat flux scalings}

Guided by the prediction (3.8) and the observations by Lepot et al. (2018) for $\operatorname{Pr}=1$ and 7 , we represent on figure 19 the Nusselt number $N u$ compensated by $\sqrt{R a}$ for data obtained in a suite of DNS runs with almost logarithmically-equispaced Prandtl number values: $\operatorname{Pr} \in\{0.003,0.01,0.03,0.1,0.3,1,3,10\}$. We observe that for a fixed Prandtl number (a given colour on the figure), the data form a plateau as the thermal forcing $R a_{Q}$ varies. This confirms that the scaling exponent $\gamma=0.5$ is universal and does not depend on the Prandtl number, in line with prediction (3.8).

We now turn to the central question of this paper - the Prandtl number dependence which amounts to analysing the height of the plateaus on figure 1 1 . On this logarithmic representation, the plateaus are separated by a distance that seems to vary with the Prandtl number.

To better characterise this effect, we turn to figure 10 where we plot the heat flux compensated by $\sqrt{R a P r}$ - the scaling prediction (3.8) in absence of boundary-layer correction - as a function of the Prandtl number. Upon this rescaling, the data points for no-slip boundary conditions (filled symbols) fall on a master curve that exhibits: (i) a plateau for $\operatorname{Pr} \lesssim 0.04$, providing evidence for the $N u \sim \sqrt{\operatorname{RaPr}}$ scaling at low Prandtl numbers; (ii) a scaling-law with slope $-1 / 3$ for $\operatorname{Pr} \gtrsim 0.04$ which, when combined with the rescaling of the figure, suggests the $N u \sim \operatorname{Ra}^{1 / 2} \operatorname{Pr}^{1 / 6}$ scaling predicted at high Prandtl numbers.

By contrast, the data points obtained for stress-free boundary conditions (open symbols) reasonably form a plateau, as they exhibit variations of less than $30 \%$ when $\mathrm{Pr}$ spans three and a half decades. This observation validates the mixing-length scaling $N u \sim \sqrt{R a P r}$ at all Prandtl numbers for stress-free boundary conditions.

To summarize, the numerical data are fully compatible with the prediction (3.8), with the following values for the dimensionless prefactors and transition Prandtl number: $c_{1}=c_{2} \simeq 0.38, c_{3} \simeq 0.13$, which leads to $P r_{*}=\left(c_{3} / c_{2}\right)^{3} \simeq 0.04$.

\subsection{Boundary layer structure: no-slip case}

We have established that, in the high-Prandtl-number regime $\operatorname{Pr} \gtrsim 0.04$, thermal transfer laws differ for a no-slip and a stress-free bottom boundary and are accurately captured by the scaling prediction (3.8). To validate a posteriori the underlying assumptions of the model, we now examine the structure of the boundary layers in the DNS flows.

The structure of the thermal boundary layer is illustrated in figure 2 for a value of the prandtl number $\operatorname{Pr}=3$ that lies comfortably within the high-Prandtl-number regime, where a clear departure is observed between no-slip and stress-free bottom boundaries. On this figure, we display the vertical temperature profiles $\bar{\theta}$, where the overline denotes an average along the horizontal directions and over time. Upon examination, the temperature profiles are strikingly similar for no-slip and stress-free in the bulk of the flow, while the no-slip case develops strong boundary layers associated with a large temperature jump at the bottom of the layer.

Beyond the mere temperature profiles, we analyse the thermal energy fluxes in the vicinity of the bottom bounding surface on figure 2(b). For no-slip boundary conditions, the diffusive flux $\overline{\partial_{z} \theta}(z)$ grows linearly with the distance from the wall $z$. As a conse- 
(a) Temperature profiles
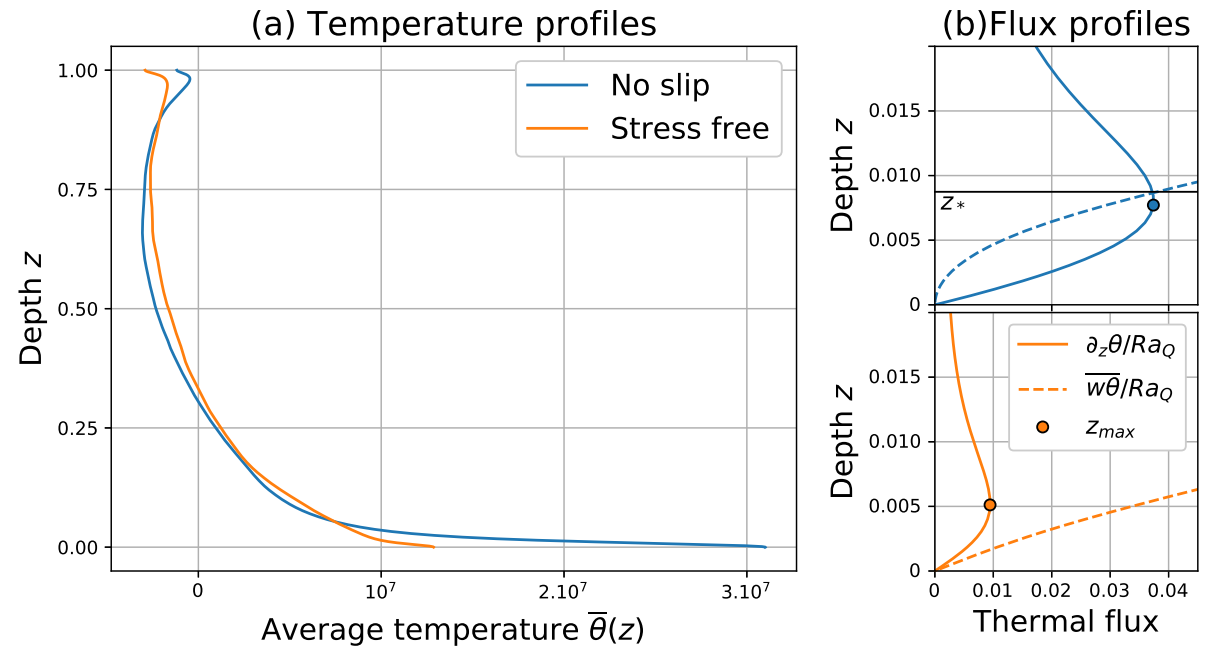

Figure 2. (a) Temperature profiles averaged in time and along horizontal directions $x$ and $y$, for $\operatorname{Pr}=3, R a_{Q}=3 \times 10^{10}$, with either a no-slip (blue) or a stress-free (orange) bottom boundary. (b) Time-and horizontally averaged contributions to the total heat-flux. Solid lines represent the diffusive flux $-\overline{\partial_{z} \theta}$, whereas dashed lines represent the convective flux $\overline{w \theta}$. Coloured circles materialise $z_{\max }$, the altitude where the diffusive flux is maximum. For a no-slip bottom boundary (top panel), the thin horizontal black line represents the altitude $z_{*}$ where the diffusive and the convective fluxes are equal (see text).

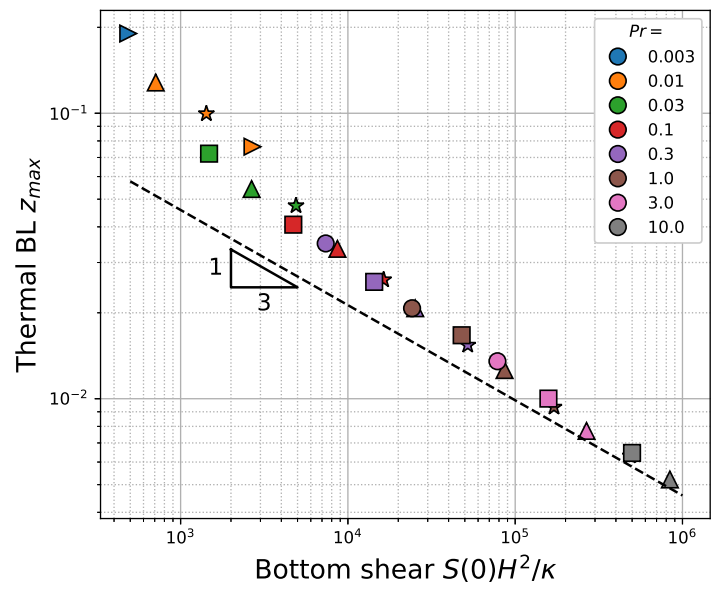

FiguRE 3. Structure of the boundary layers as $\operatorname{Pr}$ and $R a_{Q}$ vary. We represent the dimensionless thickness of the thermal boundary layer $\delta_{\Theta} / H$ defined as the depth where the diffusive flux is maximum (see figure 20 $\mathrm{b}$ ), represented as a function of the dimensionless shear at the bottom plate $S(0) H^{2} / \kappa=\overline{\partial_{z} \sqrt{u^{2}+v^{2}}}$. Dashed line: prediction from equation (3.5).

quence, the diffusive flux strongly dominates the purely convective flux $\overline{w \theta}(z)$, which exhibits a slower quadratic growth with $z$. As $z$ increases, the diffusive flux reaches a maximum at distance $z_{\max }$ and decreases again. Advection and diffusion contribute in equal proportions to the heat transfer at a comparable height $z_{*}$, where $\overline{\partial_{z} \theta}\left(z_{*}\right)=\overline{w \theta}\left(z_{*}\right)$. Above $z_{*}$ is the bulk, where pure convection proves the most efficient mechanism.

Being able to extract a thermal boundary layer thickness from the numerical solutions, we now turn our attention to the validity of the sheared boundary layer asumption (3.5) 
near a no-slip boundary. The dimensionless bottom shear $S(0) H^{2} / \kappa$ is estimated as:

$$
S(0) H^{2} / \kappa=\left.\overline{\partial_{z} \sqrt{u^{2}(x, y, z, t)+v^{2}(x, y, z, t)}}\right|_{z=0} .
$$

The boundary layer thickness measured by $z_{\max }$ is displayed on figure 3 as a function of this dimensionless bottom shear, for all the data gathered with no-slip boundary conditions. A good collapse is observed, and the data for large Prandtl number validate the shear layer structure assumed in equation (3.5). The approach to this asymptote proves rather slow, because the kinetic boundary layer is only moderately thicker than the thermal one for the highest $\operatorname{Pr}$ achieved in this study (approximately 3 times thicker for $\operatorname{Pr}=10)$.

\subsection{Boundary layer structure: stress-free case}

Returning to figure 2(b), the examination of the energy flux profiles in the stressfree case yields a much different scenario. In contrast with the no-slip case, both the convective flux $\overline{w \theta}$ and the diffusive flux $\overline{\partial_{z} \theta}$ grow linearly with the distance $z$ to the bottom boundary. As a first consequence, the ratio between these two fluxes remains asymptotically of order $O(1)$ as the distance to the wall decreases. Moreover, energy transfers are dominated by pure convection as $\overline{w \theta}>\overline{\partial_{z} \theta}$ for any altitude $z$. All these observations are in strong support of the working hypotheses used for deriving prediction (3.8), and thus shed light on the (non-purely-diffusive) nature of the boundary layers that result in the $N u \sim \sqrt{R a P r}$ scaling observed for all $\operatorname{Pr}$ on figure 1 .

\section{Discussion and conclusion}

We have characterised the dimensionless heat flux arising in convection driven by internal heat sources and sinks, focusing on the influence of the diffusivity ratio. Combining the present results with the conclusions of Lepot et al. (2018) and Bouillaut et al. (2019), we have answered the question raised at the outset - the scaling behaviour of the enhanced heat flux - by proposing the compact scaling relation (3.8) for the Nusselt number. While the scaling exponents appearing in this expression are most likely robust features of CISS, the precise values of the prefactors $c_{1}, c_{2}$ and $c_{3}$ may very well depend on details of a given system. For instance, extracting the prefactor $c_{3}$ from the experimental data obtained by Lepot et al. (2018) in a cylindrical container with a stress-free top boundary leads to approximately 0.9 . Their DNS data in a cube with stress-free sidewalls yield a value closer to $c_{3} \simeq 1.1$, while the present DNS data with periodic boundary conditions in the horizontal lead to $c_{3} \simeq 1.3$.

When a stress-free bottom boundary is considered, we have established that the heat flux follows the standard mixing-length prediction $N u \sim \sqrt{R a P r}$, both in terms of Rayleigh and Prandtl number. When the bottom boundary condition is no-slip, the mixing-length scaling regime is observed at low Prandtl number, $\mathrm{Pr} \leqslant 0.04$. As most astrophysical fluids of interest have a low-Prandtl number, this confirms that the associated flows would lead to the mixing-length scaling regime regardless of the bottom boundary condition. The present setup thus offers a clear realisation of the scaling-laws believed to hold in astrophysical contexts.

However, for $\operatorname{Pr}>0.04$ and a no-slip bottom boundary, we find that the stagnant layer in the vicinity of the latter is conducive to a modification of the Prandtl-number exponent: $N u \sim R a^{1 / 2} \operatorname{Pr}^{1 / 6}$. This scaling is well captured by modelling the bottom thermal boundary layer as a sheared layer nested inside the kinetic boundary layer. We confirmed this picture through an analysis of the temperature and heat flux profiles 
extracted from our suite of DNS. This $\operatorname{Pr}=\mathcal{O}(1)$ situation could be relevant to the atmosphere, in situations where the latter is subject to both infrared heating and cooling (Deardorff 1974). The no-slip bottom boundary and the associated stagnant layer would then crucially impact the temperature profile in the atmospheric boundary layer. But this situation is also relevant to laboratory realisations of CISS (Lepot et al. 2018; Bouillaut et al. 2019), which use water $(P r=7)$ together with a no-slip bottom boundary. Several approaches can be envisioned to achieve the $N u \sim \sqrt{\operatorname{RaPr}}$ scaling in such a laboratory experiment. Following the numerical study of Barker et al. (2014), one approach would consist in artificially picking a distribution of sources and sinks that vanishes at the top and bottom boundaries, but there is no simple experimental way of tailoring the heat source distribution. A more natural approach from the experimental standpoint would be to use a fluid with a Prandtl number much below $P r_{*}=0.04$, but that again seems rather impracticable. Finally, our study suggests a third and much more promising route towards observing the $\mathrm{Pr}^{1 / 2}$ dependence of the Nusselt number experimentally: tweaking the bottom boundary condition to make it as close as possible to a stress-free one, by stacking two layers of immiscible fluids, a heavy transparent one below a lighter light-absorbing one.

This work was performed using HPC resources from GENCI-CINES (Grant 2019A0060410803 and Grant 2020-A0082A10803). This work is supported by the European Research Council under grant agreement 757239.

Declaration of Interests. The authors report no conflict of interest.

\section{REFERENCES}

Ahlers, G., Grossmann, S. \& Lohse, D. 2009 Heat transfer and large scale dynamics in turbulent Rayleigh-Bénard convection. Rev. Mod. Phys. 81, 503-537.

Aurnou, J.M., Calkins, M.A., Cheng, J.S., Julien, K., King, E.M., Nieves, D., Soderlund, K.M. \& Stellmach, S. 2015 Rotating convective turbulence in earth and planetary cores. Phys. Earth Planet. Int. 246, $52-71$.

Aurnou, J. M., Bertin, V., Grannan, A. M., Horn, S. \& Vogt, T. 2018 Rotating thermal convection in liquid gallium: multi-modal flow, absent steady columns. J. Fluid Mech. 846, 846-876.

Barker, A. J., Dempsey, A. M. \& Lithwick, Y. 2014 Theory and simulations of rotating convection. Astrophys. J. 791 (1), 13.

Boulllaut, V., Lepot, S., Aumaître, S. \& Gallet, B. 2019 Transition to the ultimate regime in a radiatively driven convection experiment. J. Fluid Mech. 861, R5.

Browning, M. K 2008 Simulations of dynamo action in fully convective stars. Ap. J. 676 (2), 1262.

Burns, K. J., Vasil, G. M., Oishi, J. S., Leconnet, D. \& Brown, B. P. 2020 Dedalus: A flexible framework for numerical simulations with spectral methods. Phys. Rev. Research 2, 023068.

Calkins, M. A., Julien, K., Tobias, S. M. \& Aurnou, J. M. 2015 A multiscale dynamo model driven by quasi-geostrophic convection. Journal of Fluid Mechanics 780, 143-166.

Castaing, B., Gunaratne, G., Heslot, F., Kadanoff, L., Libchaber, A., Thomae, S., Wu, X.-Z., Zaleski, S. \& Zanetti, G. 1989 Scaling of hard thermal turbulence in Rayleigh-Bénard convection. J. Fluid Mech. 204, 1-30.

Chavanne, X., Chillà, F., Castaing, B., Hébral, B., Chabaud, B. \& Chaussy, J. 1997 Observation of the ultimate regime in Rayleigh-Bénard convection. Phys. Rev. Lett. 79, 3648-3651.

Chillà, F. \& Schumacher, J. 2012 New perspectives in turbulent Rayleigh-Bénard convection. Eur. Phys. J. E 35 (7), 58.

Cioni, S., Ciliberto, S. \& Sommeria, J. 1997 Strongly turbulent rayleigh-bénard convection in mercury: comparison with results at moderate prandtl number. J. Fluid Mech. 335, 111-140. 
On the role of the Prandtl number in convection driven by heat sources and sinks 11

DeARdorfF, J. W. 1974 Three-dimensional numerical study of the height and mean structure of a heated planetary boundary layer. Bound. Lay. Meteorol. 7 (1), 81-106.

Doering, C. R., Toppaladoddi, S. \& Wettlaufer, J. S. 2019 Absence of evidence for the ultimate regime in two-dimensional rayleigh-bénard convection. Phys. Rev. Lett. 123, 259401.

Fauve, S, Laroche, C \& Libchaber, A 1981 Effect of a horizontal magnetic field on convective instabilities in mercury. J. Physique Lett. 42 (21), 455-457.

Goluskin, D. 2015 Internally heated convection beneath a poor conductor. J. Fluid Mech. 771, $36-56$.

Goluskin, D. \& van Der Poel, E. P. 2016 Penetrative internally heated convection in two and three dimensions. J. Fluid Mech. 791, R6.

Guervilly, C., Cardin, P. \& Schaeffer, N. 2019 Turbulent convective length scale in planetary cores. Nature $\mathbf{5 7 0}$ (7761), 368-371.

He, X., Funfschilling, D., Nobach, H., Bodenschatz, E. \& Ahlers, G. 2012 Transition to the ultimate state of turbulent rayleigh-bénard convection. Phys. Rev. Lett. 108, 024502.

Lepot, S., Aumaître, S. \& Gallet, B. 2018 Radiative heating achieves the ultimate regime of thermal convection. Proc. Natl. Acad. Sci. 115 (36), 8937-8941.

Miquel, B., Lepot, S., Boulllaut, V. \& Gallet, B. 2019 Convection driven by internal heat sources and sinks: Heat transport beyond the mixing-length or "ultimate" scaling regime. Phys. Rev. Fluids 4, 121501.

Miquel, B., Xie, J.-H., Featherstone, N., Julien, K. \& Knobloch, E. 2018 Equatorially trapped convection in a rapidly rotating shallow shell. Phys. Rev. Fluids 3, 053801.

Niemela, J.J., Skrbek, L., Sreenivasan, K.R. \& Donnelly, R.J. 2000 Turbulent convection at very high rayleigh numbers. Nature 404 (6780), 837.

Plant, R.S. \& Yano, J.-I. 2015 Parameterization of Atmospheric Convection. Imperial College Press.

Rocha, C. B., Bossy, T., Llewellyn Smith, S. G. \& Young, W. R. 2020 Improved bounds on horizontal convection. J. Fluid Mech. 883, A41.

Shishkina, O., Emran, M. S., Grossmann, S. \& Lohse, D. 2017 Scaling relations in largeprandtl-number natural thermal convection. Phys. Rev. Fluids 2, 103502.

Shishkina, O. \& Wagner, S. 2016 Prandtl-number dependence of heat transport in laminar horizontal convection. Phys. Rev. Lett. 116, 024302.

Soderlund, K. M. 2019 Ocean dynamics of outer solar system satellites. Geophys. Res. Lett. 46 (15), 8700-8710.

Soderlund, K. M., King, E. M. \& Aurnou, J. M. 2012 The influence of magnetic fields in planetary dynamo models. Earth Planet. Sc. Lett. 333-334, 9 - 20.

Sutherland, B. R., Achatz, U., Caulfield, C. P. \& Klymak, J. M. 2019 Recent progress in modeling imbalance in the atmosphere and ocean. Phys. Rev. Fluids 4, 010501.

Vreugdenhil, C. A., Griffiths, R. W. \& Gayen, B. 2017 Geostrophic and chimney regimes in rotating horizontal convection with imposed heat flux. J. Fluid Mech. 823, 57-99.

Yano, J.-I., Talagrand, O. \& Drossard, P. 2003 Origins of atmospheric zonal winds. Nature $421,36$. 